\title{
ORIGINAL
}

\section{RIESGO DE PRESENTACIÓN DE EVENTOS CARDIOVASCULARES SEGÚN LA AGRUPACIÓN DE LOS FACTORES DE RIESGO MODIFICABLES EN LA POBLACIÓN MAYOR DE 15 DE AÑOS DE UN CENTRO DE SALUD DE BARCELONA}

José Miguel Baena Díez (1,2), José Luis del Val García (2), Laia Alemany Vilches (2), José Luis Martínez Martínez (1), Josefina Tomàs Pelegrina (1), Iván González Tejón (1), Eva María Raidó Quintana (1) y Mónica Rovira España (1)

(1) Área Básica de Salud (ABS) Dr. Carles Ribas. La Marina. Barcelona.

(2) UI SAP Sants-Montjuïc-Sarrià-Les Corts-Sant Gervasi.

\section{RESUMEN}

Fundamento: En la últimas décadas la mortalidad por enfermedades cardiovasculares ha mostrado una tendencia decreciente en los países desarrollados, confirmada asimismo en España. No obstante siguen siendo la principal causa de mortalidad El objetivo de este trabajo es estudiar la asociación entre las enfermedades cardiovasculares y diversos factores de riesgo cardiovascular modificables en relación a su agrupación (clustering).

Métodos: Estudio descriptivo transversal realizado en un centro de salud urbano, que incluyó a 2.248 personas de 15 o más años, seleccionadas por muestreo aleatorio simple del archivo de historias clínicas. Se calculó en forma de odds ratio (OR) el riesgo de tener alguna enfermedad cardiovascular (cardiopatía isquémica, enfermedad cerebrovascular o arteriopatia periférica de extremidades inferiores) en relación al clustering de los factores de riesgo tabaquismo, hipertensión arterial, hipercolesterolemia, hipertrigliceridemia y diabetes mellitus, ajustado por edad, sexo y factores de riesgo.

Resultados: Las personas estudiadas tenían 224 enfermedades cardiovasculares. En el tabaquismo la OR como factor de riesgo aislado fue de 1,5 (IC95\%: 1,0-2,2) y de 1,6 (IC95\%: 0,9-2,5) con el clustering con los otros 4 factores de riesgo; con la hipertensión arterial de 2,1 (IC95\%: 1,5-2,9) y de 1,7 (IC95\%: 1,1-2,6), respectivamente; con la hipercolesterolemia de 1,7 (IC95\%: 1,2-2,4) y de 1,6 (IC95\%: 1,1$2,4)$, respectivamente; con la diabetes de 2,5 (IC95\%: $1,7-3,5$ ) y 2,0 (IC95\%: 1,3-3,0), respectivamente y con la hipertrigliceridemia de 1,8 (IC95\%: 1,2-2,8) y 1,3 (IC95\%: 0,8-2,1), respectivamente. Las OR se comportaron de manera similar al estratificar por cada enfermedad cardiovascular, aunque las OR más elevadas (entre 2,4 y 3,1) correspondieron al clustering de diabetes mellitus y tabaquismo.

Conclusiones: El riesgo de tener enfermedades cardiovasculare sigue siendo elevado con el clustering de factores de riesgo cardiovascular, aunque se observan diferencias entre ellos.

Palabras clave: Enfermedades cardiovasculares. Factores de riesgo. Cardiopatía isquémica. Accidente cerebrovascular. Enfermedades vasculares periféricas. Análisis por conglomerados. Agrupamientos.

Correspondencia:

José Miguel Baena Díez.

C/ Sant Joan, 123. Escalera 2, $3^{\circ} 2^{\mathrm{a}}$

Parets del Vallès

08150 Barcelona

Correo electrónico: jmbaena.pbcn@ics.scs.es

\section{ABSTRACT}

\section{Risk of Suffering from Cardiovascular Diseases because of the Clustering of the Modifiable Cardiovascular Risk Factors in the Population Older than 15 years of a Health Care Center in Barcelona}

Background: Over recent decades, the death rate due to cardiovascular diseases has shown a downward trend in developed countries, as has also been the case in Spain. However, are still the leading cause of death. This study is aimed at studying the relationship between cardiovascular diseases and different modifiable cardiovascular risk factors related to their clustering.

Methods: Descriptive cross-sectional study conducted at an urban healthcare center, which included 2248 individuals ages 15 and above selected by simple random sampling of the medical record files. The risk of having some cardiovascular disease (ischemic cardiopathy, cerebrovascular disease or peripheral arteriopathy of lower limbs) was calculated in the form of an odds ration (OR) in relation to the clustering of the risk factors of smoking, high blood pressure, hypercholesterolemia, hypertriglyceridemia and diabetes mellitus, adjusted by age, sex and risk factors.

Results: The individuals studied had 224 cardiovascular diseases. For smoking, the OR as an isolated risk factor was $1.5(95 \% \mathrm{CI}$ : 1.0-2.2) and 1.6 (95\% CI: 0.9-2.5) with the clustering with the other four risk factors; with high blood pressure, respectively of $2.1(95 \%$ CI: $1.5-2.9)$ and 1.7 (95\% CI: 1.1-2.6); with hypercholesterolemia, respectively of 1.7 (95\% CI: 1.2-2.4) and 1.6 (95\% CI: 1.1-2.4); and with hypertriglyceridemia, respectively of $1.8(95 \% \mathrm{CI}: 1.2-2.8)$ and 1.3 (95\% CI: $0.8-2.1)$. The OR's showed a similar behavior on layering by each cardiovascular disease, although the highest OR's (2.4 3.1 range) corresponded to the clustering of diabetes mellitus and smoking.

Conclusions: The risk of having cardiovascular diseases remains high with the clustering of cardiovascular risk factors, although differences among them are found to exist.

Key words: Cardiovascular diseases. Risk factors. Coronary heart disease. Cerebrovascular accident. Peripheral angiopathies. Clustering. 


\section{INTRODUCCIÓN}

En la últimas décadas la mortalidad por enfermedades cardiovasculares ha mostrado una tendencia decreciente en los países desarrollados, confirmada asimismo en Espa$\tilde{\text { na }}{ }^{1}$. No obstante, las enfermedades cardiovasculares siguen siendo la principal causa de mortalidad, produciendo el $40 \%$ de la misma en nuestro medio ${ }^{2}$, destacando por su impacto la cardiopatía isquémica y la enfermedad cerebrovascular ${ }^{1-3}$.

Las enfermedades cardiovasculares tienen un origen multifactorial, siendo claves en su etiopatogenia los factores de riesgo cardiovascular, tanto los no modificables (como la edad y el sexo) como los denominados modificables (FRCVM), entre los que podemos destacar el tabaquismo, la hipertensión arterial, la hipercolesterolemia y la diabetes mellitus ${ }^{4}$. Otros FRCVM, como la hipertrigliceridemia ${ }^{5}$ y la obesidad ${ }^{6}$, también pueden tener un papel relevante. Sin embargo, en los países mediterráneos las tasas de cardiopatía isquémica son muy inferiores a las de los países del norte de Europa, con un similar o incluso mejor perfil de FRCVM clásicos $^{7,8}$. Parece por tanto que el impacto de dichos FRCVM sobre la aparición de eventos cardiovasculares mayores (ECV) es muy diferente de unos a otros países con niveles similares de desarrollo ${ }^{7,8}$. Esta hipótesis también se ha confirmado recientemente en nuestro país por parte del grupo REGICOR, que muestra bajas tasas de enfermedad coronaria a pesar de la elevada prevalencia de FRCVM ${ }^{9}$.

Aunque el impacto de cada uno de los FRCVM reseñados sobre el riesgo de ECV está bien estudiado ${ }^{4-6}$, son escasos los estudios que han abordado el papel de la agrupación (clustering) de los FRCVM ${ }^{10-11}$. En un estudio previo de nuestro grupo ${ }^{12}$ se investigó en nuestro medio este fenómeno, con resultados similares a los trabajos reseñados ${ }^{10-11}$, de manera que el clustering de FRCVM se acompañó de un mayor riesgo de ECV. Sin embargo no conocemos bien el efecto del incremento o adición de dichos FRCVM de manera individual sobre el riesgo de ECV. Por ejemplo, ¿es lo mismo tener hipercolesterolemia que hipertensión cuando además hay otros FRCVM en relación al riesgo de tener ECV? Asimismo, tampoco conocemos bien el efecto de los FRCVM en relación a su clustering tras ajustar por el resto de FRCVM.

El objetivo del presente trabajo es estudiar el impacto de cada FRCVM sobre el riesgo de presentar algún ECV (cardiopatía isquémica, enfermedad cerebrovascular o arteriopatía periférica de extremidades inferiores) en relación al clustering con los otros FRCVM en un medio como el nuestro de baja prevalencia de ECV.

\section{SUJETOS Y MÉTODOS}

Recogida de los datos: Se realizó un diseño descriptivo transversal. El ámbito del estudio fue un Centro de Salud urbano, situado en un barrio periférico de la ciudad de Barcelona, con una población bastante heterogénea respecto al nivel socioeconómico, abarcando desde zonas marginales a zonas de clase media. Otra característica importante es la fácil accesibilidad de los usuarios y la elevada frecuentación. En el momento de realizarse el estudio constaban en el archivo del centro 35.277 historias clínicas (HC) y trabajaban 16 médicos de familia. Los participantes fueron seleccionados por muestreo aleatorio simple del archivo de $\mathrm{HC}$, incluyéndose a las personas con edad igual o superior a 15 años, un mínimo de 5 visitas registradas en la $\mathrm{HC}$ y al menos 3 visitas en los últimos dos años. Si la $\mathrm{HC}$ seleccionada no cumplía estos criterios se extraía una nueva de manera consecutiva al número aleatorio seleccionado. La información se recogió desde junio de 1998 a mayo de 1999 a partir de la revisión de la HC mediante un protocolo estandarizado, en el que se recogieron, además de la edad en años 
y el sexo, diversos FRCVM y ECV. Previamente se realizó una prueba piloto con 20 casos, para detectar errores de diseño, entrenar a los médicos que realizaron la revisión de las HC (un total de 12 médicos, todos ellos del Centro de Salud) y comprobar el grado de concordancia respecto a las variables de estudio entre los encuestadores y un gold standard consensuado. Con el objeto de disminuir la proporción de no respuestas mientras se recogió la información se contactó con los médicos responsables de los pacientes en cuyas HC no constaban la totalidad de los FRCVM estudiados y telefónicamente con 418 pacientes en los que no constaba el consumo de tabaco con los criterios descritos a continuación, con un mínimo de 3 llamadas en tres franjas horarias diferentes (mañana, tarde y noche) y al menos una llamada en sábado o domingo.

\section{Factores de riesgo cardiovascular modificables estudiados (FRCVM)}

Se registraron los siguientes FRCVM, con la consideración de que en las personas con ECV los FRCVM estudiados sólo se consideraron si estaban presentes antes del ECV:

1) Tabaquismo. Se codificó dicha variable de manera dicotómica en fumador, no fumador o ex-fumador (más de 12 meses sin fumar), siempre que constase el consumo de tabaco en la HC durante los dos últimos años ${ }^{13}$.

2) Hipertensión arterial ${ }^{14}$. Se clasificó a los pacientes en hipertensos (3 determinaciones iguales o superiores a 140/90 mm Hg en tres visitas consecutivas) y no hipertensos, teniéndose en cuenta las tomas de tensión arterial durante los últimos 4 años si el paciente tenía entre 15 y 40 años y durante los dos últimos años si tenía más de 40 años ${ }^{14}$.

3) Hipercolesterolemia. Se codificó como variable dicotómica (si/no), en fun- ción de un punto de corte de $250 \mathrm{mg} / \mathrm{dl}$ (al menos en dos ocasiones, válidas si constaban en la HC durante los últimos 6 años ${ }^{14}$ ).

4) Hipertrigliceridemia. También codificada dicotómicamente en función de un punto de corte de $200 \mathrm{mg} / \mathrm{dl}$ (al menos dos determinaciones, válidas también si constaban en la $\mathrm{HC}$ durante los últimos 6 años ${ }^{14}$ ).

5) Obesidad. Se definió a partir de un índice de masa corporal (IMC) igual o superior a 30, producto de dividir el peso en $\mathrm{Kg}$ por la talla en metros elevada al cuadrado ${ }^{13}$. Para ello se recogieron las variables talla en metros (último valor registrado en la $\mathrm{HC}$ si el paciente era de edad superior a 18 años y el valor del último año si la edad era entre 15 y 18 años) y peso en kilogramos (se tuvo en cuenta el valor máximo registrado en la $\mathrm{HC}$, siempre que constase en los últimos 4 años).

6) Diabetes melllitus. Se utilizó la definición de la Asociación Americana de Diabetes y la OMS, adoptada por la Sociedad Española de Medicina Familiar y Comunitaria $^{15}$ : clínica típica más una glucemia al azar superior a $200 \mathrm{mg} / \mathrm{dl}$, dos glucemias basales en plasma iguales o superiores a $126 \mathrm{mg} / \mathrm{dl}$ ó test de tolerancia oral a la glucosa a las 2 horas igual o superior a $200 \mathrm{mg} / \mathrm{dl}$. También se incluyeron los casos diagnosticados fuera del Centro de Salud o que ya se trataban con insulina o antidiabéticos orales. Sólo se tuvieron en cuenta las glucemias basales en plasma (GBP) de los últimos tres años ${ }^{15}$.

Eventos cardiovasculares (ECV): $\mathrm{Se}$ estudió el número de ECV y su tipo, registrados en cualquier momento de la $\mathrm{HC}$, considerando como tales a los siguientes:

1) Cardiopatía isquémica: diagnóstico de infarto agudo de miocardio o angina de pecho, demostrados mediante electrocardiograma, curva enzimática, gammagrafía o coronariografía. 
2) Enfermedad cerebrovascular: diagnóstico clínico en informes hospitalarios de accidente isquémico transitorio (con TAC o RMN normal) o accidente cerebrovascular establecido confirmado por TAC o RMN.

3) Arteriopatía periférica de extremidades inferiores: diagnóstico clínico explícito en historia clínica o informes hospitalarios de claudicación intermitente, gangrena o úlceras isquémicas o arteriografía o eco doppler diagnósticas.

Análisis estadístico: Se realizó mediante el paquete SPSS. El cálculo del tamaño muestral se detalla en el trabajo previo reseñado anteriormente ${ }^{12}$. La concordancia en la prueba piloto se estudió mediante el estadístico kappa, considerándose que valores superiores a 0,80 eran indicativos de una buena concordancia. Las proporciones se compararon mediante la prueba de chi al cuadrado. El impacto de cada FRCVM sobre el riesgo de presentar algún ECV (cardiopatía isquémica, enfermedad cerebrovascular o arteriopatía periférica de extremidades inferiores) en relación al clustering con los otros FRCVM se estudió mediante la odds ratio (OR). En el caso de la obesidad el elevado número de no respuestas desestimó incluirla como FRCVM en los análisis, puesto que el número de sujetos incluidos en los modelos de regresión logística sería bastante inferior al $50 \%$ del total de sujetos estudiados. El ajuste de la OR se realizó mediante regresión logística tras verificar sus condiciones de aplicación. En primer lugar se calculó la OR ajustada por edad y sexo de cada FRCVM aislado. Con posterioridad de calcularon mediante modelos de regresión logística dichas OR para cada FRCVM con el clustering con cada uno de los otros FRCVM, con el clustering con las posibles combinaciones de 2 y 3 FRCVM, y finalmente con el clustering con los otros 4 FRCVM, ajustando las OR por edad, sexo y el resto de FRCVM. Se adoptó un nivel alfa de significación estadística inferior a 0,05 en todos los casos.

\section{RESULTADOS}

Se estudió un total de 2.248 personas, con una edad media de 49,1 años (DE 18,9) y un $53,5 \%$ de mujeres. El estudio de la concordancia de los encuestadores (índice kappa) dio como resultado valores superiores a 0,80 en todas las variables de estudio.

En la tabla 1 se detallan los FRCVM de las personas estudiadas. La proporción de no respuestas se detalla en la tabla 1 . Se halló una proporción de registro inferior $(\mathrm{p}<0,05)$ en los FRCVM correspondientes a hipertensión arterial, hipercolesterolemia, hipertrigliceridemia y diabetes mellitus en los pacientes que no tenían los ECV estudiados respecto a los que sí tenían registrados ECV. En cuanto al número de FRCVM no tenían ninguno registrado 879 pacientes $(39,1 \%)$, 737 (32,8\%) tenían 1 FRCVM, 394 (17,5\%) tenían 2 FRCVM, $155(6,9 \%)$ tenían 3 FRCVM y 83 (3,7\%) más de 3 FRCVM.

Un total de 224 pacientes $(10,0 \%)$ habían tenido algún ECV. 123 (5,5\%) tenían cardiopatía isquémica, $84(3,7 \%)$ enfermedad cerebrovascular y $55(2,4 \%)$ arteriopatía periférica de extremidades inferiores. La distribución por edades se detalla en la tabla 2, observándose que en la mayor parte de los casos la prevalencia se concentraba en los mayores de 65 años. Tenían un sólo ECV registrado 192 casos $(8,5 \%)$, mientras que $28(1,3 \%)$ tenían 2 ECV y 4 tenían 3 ECV $(0,2 \%)$.

En la tabla 3 se detalla la magnitud del efecto en forma de OR de cada FRCVM estudiado de manera aislada (1 FRCVM) y en relación con el clustering de 2 FRCVM, 3 FRCVM y 4 FRCVM respecto al riesgo de padecer algún ECV. Se observa que como FRCVM aislado la mayor magnitud del efecto correspondió a la diabetes mellitus y la hipertensión arterial. El tabaquismo mantuvo la magnitud de la OR con la adición progresiva de FRCVM, con valores entre 1,4 y 1,6. La hipertensión arterial mantuvo 
Tabla 1

Factores de riesgo cardiovascular modificables en los 2248 pacientes estudiados

\begin{tabular}{|l|c|c|c|}
\hline & SI & NO & No respuestas \\
& $\mathbf{n}(\mathbf{\%})$ & $\mathbf{n}(\mathbf{\%})$ & $\mathbf{n})$ \\
\hline Tabaquismo & $570(35,2)$ & $1050(64,8)$ & $628(27,9)$ \\
Hipertensión arterial & $620(33,7)$ & $1219(66,3)$ & $409(18,2)$ \\
Hipercolesterolemia & $379(21,9)$ & $1352(78,1)$ & $517(23,0)$ \\
Hipertrigliceridemia & $182(12,7)$ & $1253(87,3)$ & $813(36,2)$ \\
Obesidad & $328(32,7)$ & $676(67,3)$ & $1244(55,3)$ \\
Diabetes mellitus & $265(15,8)$ & $1412(84,2)$ & $571(25,4)$ \\
\hline
\end{tabular}

Tabla 2

Distribución por edad de los eventos cardiovasculares estudiados

\begin{tabular}{|l|c|}
\hline Factor de riesgo & $\mathbf{n}(\mathbf{\%})$ \\
\hline Algún evento cardiovascular & $12(5,3)$ \\
$15-44$ & $59(26,4)$ \\
$45-64$ & $153(68,3)$ \\
$>64$ & \\
Cardiopatía isquémica & $7(5,7)$ \\
$15-44$ & $28(22,8)$ \\
$45-64$ & $88(71,5)$ \\
$>64$ & \\
Enfermedad cerebrovascular & $0(0)$ \\
$15-44$ & $23(27,4)$ \\
$45-64$ & $61(72,6)$ \\
$>64$ & $4(7,3)$ \\
Arteriopatía periférica de extremidades inferiores & $18(37,7)$ \\
$15-44$ & $33(60,0)$ \\
$45-64$ & \\
$>64$ & \\
\hline
\end{tabular}

valores similares de la OR con el clustering de 1 FRCVM (entre 1,9 y 2,1) y tuvo una tendencia a disminuir ligeramente con el clustering de 2, 3 y 4 FRCVM. La hipercolesterolemia tuvo una menor magnitud del efecto como FRCVM aislado (OR de 1,7) pero dicho efecto se mantuvo alrededor de valores entre 1,5 y 1,7 con el clustering con los otros FRCVM. En el caso de la diabetes mellitus sucedió algo similar a la hiperten- sión arterial, aunque los valores de la OR oscilaron bastante con la asociación con 1 FRCVM, desde una OR de 1,9 asociada a la hipertrigliceridemia hasta un valor de 2,7 con el tabaquismo. Por último, con la hipertrigliceridemia la OR fue disminuyendo de manera progresiva con la adición del resto de FRCVM, alcanzando finalmente un valor de 1,3 con el clustering con 4 FRCVM. 


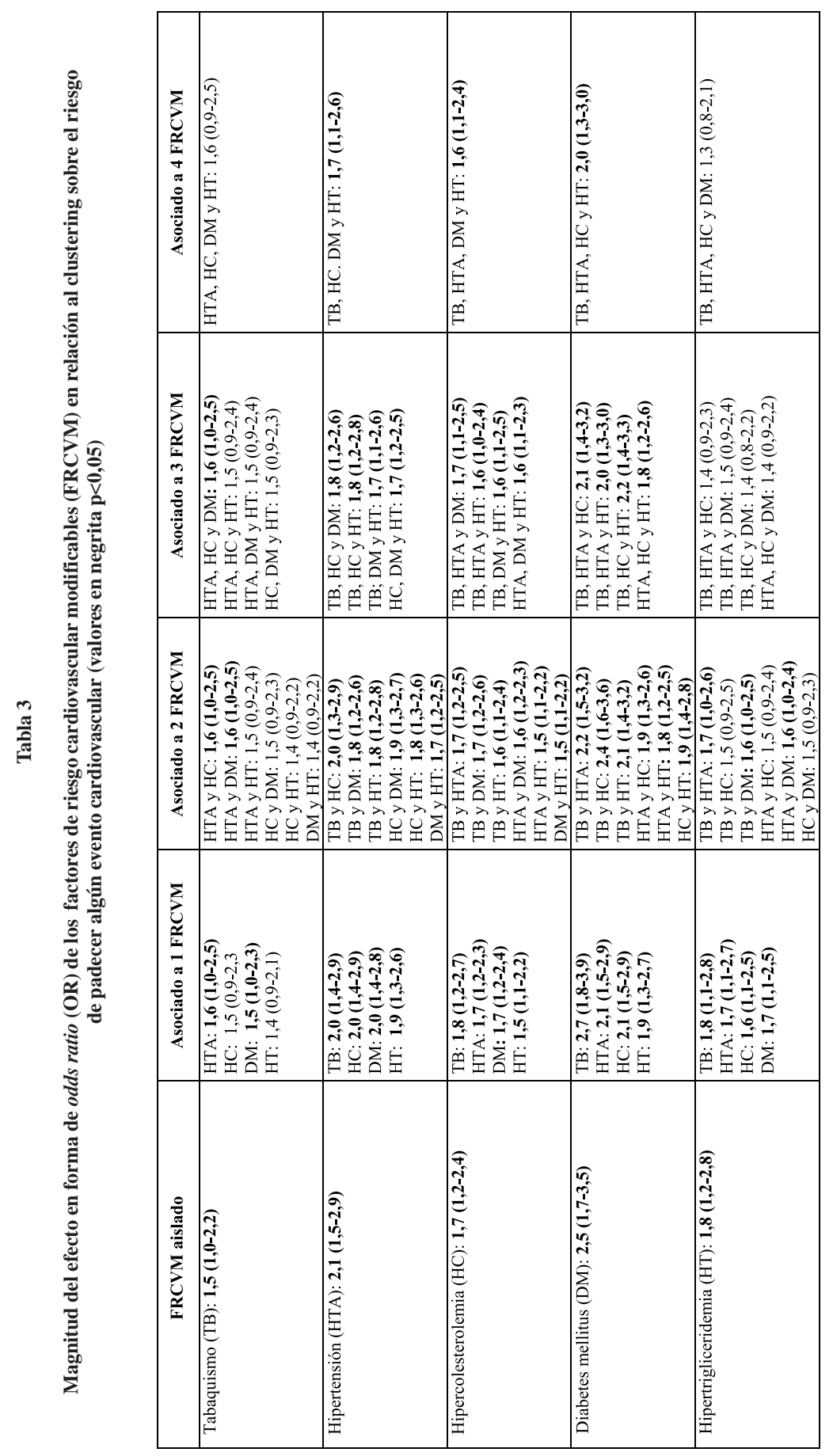




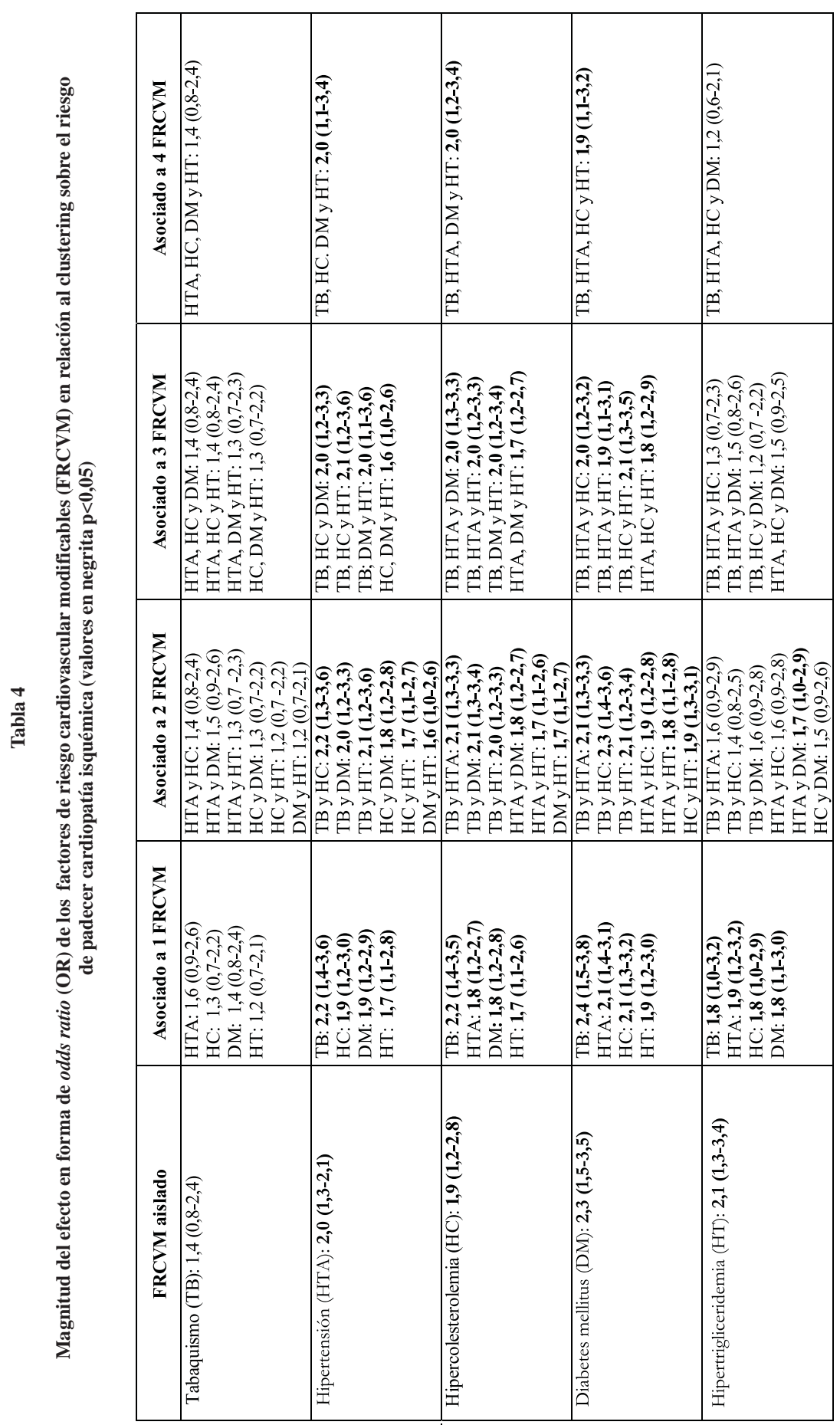




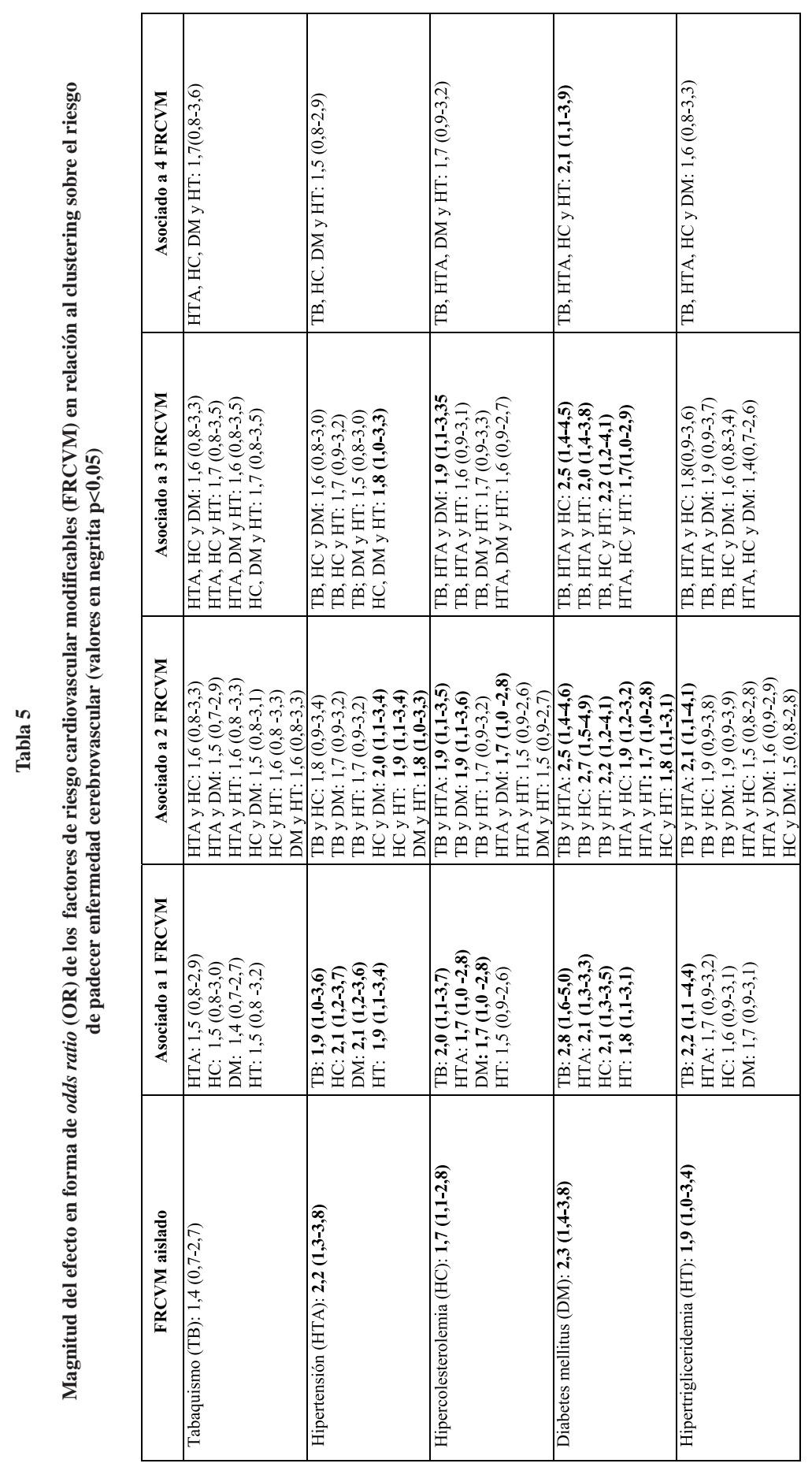




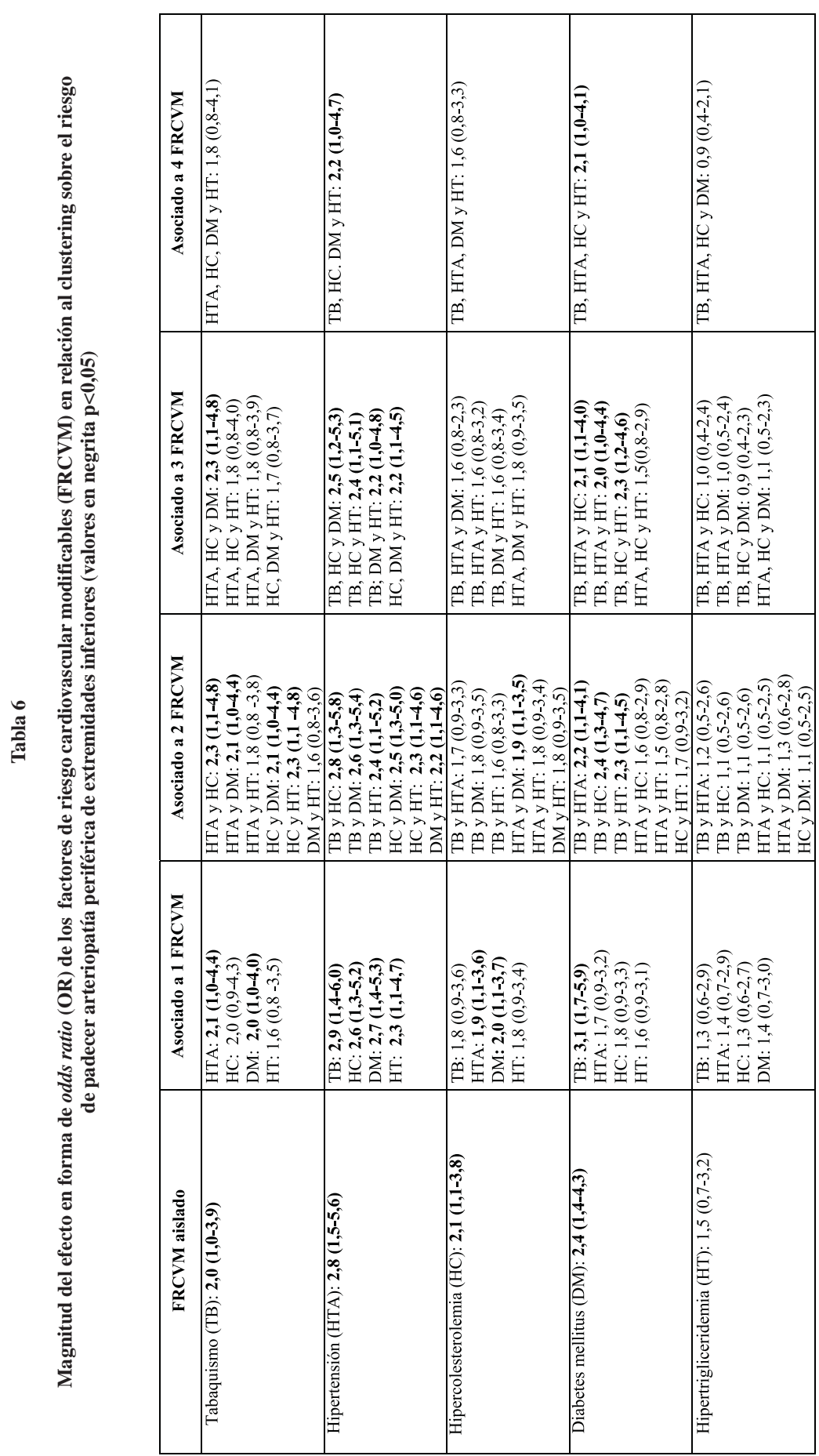


En las tablas 4-6 se presentan los mismos resultados de la tabla 3 detallados para cada ECV estudiado (cardiopatía isquémica, enfermedad cerebrovascular y arteriopatía periférica de las extremidades inferiores, respectivamente). Así, en la tabla 4 (cardiopatía isquémica) se observa que el tabaquismo se comporta de manera similar al caso anterior, que la hipertensión arterial, la hipercolesterolemia y, en menor medida, la diabetes mellitus, tienden a mantener la magnitud de la OR con el clustering de FRCVM. Sin embargo la hipertrigliceridemia pasa de una OR de 2,1 (FRCVM aislado) a 1,2 con el clustering con los otros 4 FRCVM. En la enfermedad cerebrovascular (tabla 5), el tabaquismo tiende a aumentar la magnitud del efecto, la hipertensión a disminuir las OR con el clustering, la hipercolesterolemia mantiene los valores de la OR, la diabetes mellitus también (aunque de nuevo las OR son bastante más elevadas asociadas al tabaquismo) y la hipertrigliceridemia de nuevo tiende a disminuir la magnitud de efecto. Por ultimo, en la arteriopatía periférica de las extremidades inferiores destacaron como FRCVM el tabaquismo, aunque presentó valores bastante dispares según el FRCVM al que se asociaba, la hipertensión arterial, la hipercolesterolemia (en esta caso la magnitud de la OR disminuyó bastante con el clustering) y la diabetes mellitus, sobre todo cuando se asociaba al tabaquismo, aunque en todos los caso hubo una tendencia a la disminución de las OR con el clustering. De manera similar a los casos anteriores, la hipertrigliceridemia disminuyó de manera progresiva su efecto con la adición de los otros FRCVM, hasta valores alrededor de 1 con el clustering de más de 2 FRCVM.

\section{DISCUSIÓN}

En el presente trabajo el riesgo de padecer ECV expresado en forma de OR de los FRCVM estudiados se ha mantenido, en general, con el clustering con los otros
FRCVM. La hipertensión arterial, la hipercolesterolemia y la diabetes mellitus han sido los FRCVM más importantes en relación al riesgo de padecer ECV. El riesgo ha sido especialmente elevado con la diabetes mellitus asociada al tabaquismo y de escasa cuantía en el caso de la hipertrigliceridemia con el clustering con los otros FRCVM.

En cuanto a las limitaciones de la investigación en primer lugar pueden existir sesgos de selección. A pesar de la amplia cobertura del centro de salud (el número de historias clínicas es superior a la población censada) no podemos estrictamente hablar de un estudio poblacional al tratarse de población demandante y con visitas registradas. En segundo lugar, pueden existir sesgos de información. Para evitarlos se realizó la prueba piloto para entrenar a los encuestadores y verificar la concordancia de los mismos, con el resultado de valores del índice kappa superiores a 0,80 en todos los casos. Se trató de minimizar las pérdidas de información mediante el contacto con el médico responsable de cada paciente y mediante entrevista telefónica en el caso del tabaquismo. En relación a las no respuestas se optó por no incluir en los análisis la obesidad, porque posiblemente su estimación estaba sesgada (se registra más el peso y la talla en los sujetos con obesidad evidente) y el número de sujetos incluidos en los modelos de regresión logística habría sido excesivamente bajo, puesto que sólo se incluyen los casos con todos los factores de riesgo. El número de sujetos incluidos en los análisis de las tablas 3-6 osciló entre $81,8 \%$ y el $55,6 \%$, aunque estuvo muy influenciado al incluir la hipertrigliceridemia, FRCVM con más proporción de no respuestas. Asimismo, pensamos que las no respuestas pueden afectar más a los FRCVM que a los ECV estudiados, ya que es poco probable que estos últimos no se detallen en la historia clínica. Asimismo, la superior proporción de no respuestas en el registro de los FRCVM en los casos sin ECV puede haber ocasionado una sobreestimación del efecto. Sin embargo, la 
proporción de no respuestas fue razonable en la mayor parte de FRCVM. Como en cualquier tipo de estudio, pueden aparecer fenómenos de confusión, por lo que se procedió a ajustar las OR por edad y sexo y el resto de FRCVM. No obstante no pudimos ajustar el efecto por otros FRCVM como la obesidad (por los motivos ya comentados) o el sedentarismo, debido a su bajo registro en las historias clínicas. Otra limitación es la clasificación de los FRCVM de manera dicotómica, ya que mayor intensidad del FRCVM mayor riesgo de desarrollar ECV, pero tener en cuenta este factor habría complicado sobremanera los cálculos, además de suponer una pérdida de potencia estadística. Por último, al ser un estudio descriptivo transversal es más difícil atribuir relaciones causales, aunque creemos que el requisito de exigir que los FRCVM fuesen anteriores a los ECV registrados y el hecho de que está claramente demostrada la asociación etiológica entre los FRCVM y los $\mathrm{ECV}^{4-6}$ tienden a minimizar esta limitación.

La mayoría de los pacientes $(60,9 \%)$ tenían algún FRCVM y el 28,1\% tenían dos o más FRCVM. Por tanto nuestro estudio es coincidente con el del grupo REGICOR realizado en la provincia de Gerona ${ }^{9}$. Asimismo, la proporción de pacientes con enfermedad cerebrovascular y, sobre todo, cardiopatía isquémica, es muy inferior a otros trabajos ${ }^{10-11}$ que han estudiado el efecto del clustering de FRCVM fuera de nuestro medio, a pesar de no diferir excesivamente en el perfil de FRCVM. Se confirma por tanto el bajo riesgo cardiovascular en nuestro medio ${ }^{9}$.

Los trabajos previos ${ }^{10-12}$ que habían estudiado el efecto del clustering de los FRCVM respecto al riesgo de padecer ECV habían establecido que dicho riesgo aumentaba de manera progresiva con la adición de FRCVM. Sin embargo no habían estudiado el papel individual de cada FRCVM ni habían ajustado los riesgos en función del resto de FRCVM (sólo lo habían hecho por edad y sexo, básicamente) por lo que no era de extrañar que el clustering de FRCVM se acompañase del aumento progresivo del riesgo. La aportación de nuestro trabajo consiste en sugerir que, a pesar del ajuste por edad, sexo y FRCVM, los FRCVM estudiados siguen manteniendo una magnitud del efecto relevante y que no todos ellos ni todas las asociaciones de FRCVM se comportan exactamente igual, con la excepción de la hipertrigliceridemia. En este sentido, las diferentes tablas de riesgo cardiovascular nos dan una idea global del clustering de los factores FRCVM además de la edad y el sexo, de manera que a mayor clustering y mayor intensidad de los FRCVM mayor $\mathrm{RCV}$, aunque sin precisar de manera explícita como en el presente trabajo el papel individual de cada FRCVM respecto al riesgo de ECV.

En el caso de los FRCVM estudiados todos se comportaron como factores de riesgo, aunque frecuentemente los valores no alcanzaron significación estadística por un problema de potencia estadística. No obstante, el diseño utilizado no permite atribuir relaciones causales, por lo que estas conclusiones deben de interpretarse con cautela. El tabaquismo destacó como FRCVM en el caso de la arteriopatía periférica y la magnitud de su efecto fue inferior en los otros ECV. Este resultado puede estar influido por el hecho de que la proporción de pacientes con cardiopatía isquémica y enfermedad cerebrovascular fue superior en los de edad avanzada (tabla 2), con prevalencias mucho más bajas de consumo de tabaco. En el caso de la hipertrigliceridemia se observó una cierta tendencia, pero posiblemente parte del efecto sea por su asociación a la diabetes mellitus y el sexo masculino. La hipertensión, la hipercolesterolemia y sobre todo la diabetes mellitus (especialmente al asociarse al consumo de tabaco) fueron los FRCVM con mayores OR y que menos fluctuaron con el ajuste.

Las políticas sanitarias se deben de encaminar hacia la reducción de ECV, hecho que 
puede lograrse actuando a diferentes niveles. En primer lugar a través de la prevención primaria de los FRCVM ${ }^{14}$. Para ello es básica la adopción de estilos de vida que prevengan su aparición. Por ejemplo estimulando el ejercicio físico, la pérdida de peso en los casos de obesidad o sobrepeso y actuando sobre el consumo de tabaco y el consumo excesivo de alcohol. Estas actuaciones son especialmente efectivas si las intervenciones son multifactoriales ${ }^{16}$. La adopción de estos estilos de vida saludables no sólo previene la aparición de los FRCVM más importantes sino que además disminuye la morbimortalidad cardiovascular. Por ejemplo, se ha comprobado que el ejercicio físico disminuye la incidencia de accidentes cerebrovasculares y de cardiopatía isquémica ${ }^{17-19}$, Similares consideraciones pueden hacerse con la pérdida de peso o el abandono del hábito tabáquico $^{20}$. En segundo lugar, pueden reducirse los ECV a través del control de los FRCVM. En este sentido, está claramente demostrada mediante metaanálisis la reducción de ECV al reducir los niveles de colesterol y tensión arterial $^{21-22}$, aunque el control de los FRCVM mediante dieta y/o fármacos no logra disminuir el riesgo de cardiopatía isquémica y enfermedad cerebrovascular por debajo del que tienen los pacientes sin dichos FRCVM ${ }^{10}$, hecho que reafirma la importancia de la prevención primaria, especialmente a partir de los médicos de fami$\mathrm{lia}^{23}$, como en el caso del Programa de Actividades Preventivas y Promoción de la Salud $^{14}$ (PAPPS) de la Sociedad Española de Medicina Familiar y Comunitaria (SEMFYC). Sin embargo, éste y otros programas deberían complementarse con otras actuaciones a nivel comunitario ${ }^{24-25}$. En este sentido, es importante remarcar que en los últimos años se ha producido un cambio favorable en el perfil de los FRCVM en los países desarrollados ${ }^{26}$, habiendo un descenso del consumo de tabaco, de la tensión arterial y de los niveles de colesterol, aunque con un aumento del índice de masa corporal y de la prevalencia de diabetes mellitus, confirmada asimismo en el presente trabajo.
Es preciso abordar una estrategia global de prevención de los ECV a través de la prevención, control y tratamiento de los FRCVM, con el objetivo de reducir la incidencia de $\mathrm{ECV}^{10,26}$. En este sentido se acepta que el riesgo de padecer ECV debe de valorarse globalmente a partir de la utilización de tablas de riesgo cardiovascular, como las de Framingham, utilizadas hasta ahora $^{27}$, las calibradas por el grupo REGI$\mathrm{COR}^{9}$, o más recientemente las del grupo $\mathrm{SCORE}^{28}$, a pesar de las limitaciones que tienen, entre las que podemos destacar que valoran (Framingham, REGICOR) básicamente el riesgo coronario (aunque sea teóricamente una buena aproximación al riesgo cardiovascular), que sobreestiman el riesgo (Framingham) y que acaban a los 65 años y sólo valoran mortalidad (SCORE), aunque la mayor parte de los ECV se darán a partir de los 65 años y frecuentemente no serán mortales. Sin embargo persisten las dudas, especialmente a la hora de decidir un tratamiento hipolipemiente, cuando el riesgo cardiovascular es bajo pero existen FRCVM importantes o con valores extremos. En este sentido, el grupo REGICOR ${ }^{9}$ aconseja acertadamente tratar la hipertensión independientemente del valor de riesgo cardiovascular, ya que el beneficio se extiende a otros ECV y no sólo a la cardiopatía isquémica. En el caso de la diabetes mellitus parece bastante claro que el control metabólico y sobre todo el tratamiento con metformina ${ }^{29}$ mejoran las complicaciones macrovasculares y, por tanto, debería tratarse siempre, ya que además evita las descompensaciones cetoacidóticas. Por último en relación con la hipercolesterolemia, los expertos españoles aconsejan tratarla también en función del riesgo cardiovascular $^{2}$, aunque recientemente en el estudio ALLHAT $^{30}$ el tratamiento hipolipemiante no produjo beneficios en los pacientes con hipercolesterolemia e hipertensión arterial.

\section{BIBLIOGRAFÍA}

1. Villar Álvarez F, Banegas Banegas JR, Rodríguez Artalejo F, del Rey Calero J. Mortalidad cardio- 
vascular en España y sus comunidades autónomas (1975-1992). Med Clin (Barc) 1998; 110: $321-27$.

2. Ministerio de Sanidad y Consumo, Sociedad Española de Cardiología y Sociedad Española de Arteriosclerosis. Control de la colesterolemia en España, 2000. Un instrumento para la prevención cardiovascular. Rev Esp Salud Pública 2000; 74: 215 53.

3. Olalla MT, Medrano MJ, Sierra MJ, Almazán J. Time trends, cohort effect and spatial distribution of cerrebrovascular disease mortality in Spain. Eur J Epidemiol 1999; 15: 331-39.

4. Anderson KM, Wilson PWF, Odell PM, Kannel WB. An Updated coronary risk profile. A Statement for health professionals. Circulation 1991; 83: 356-62.

5. Jeppesen J, Hein HO, Suadicani P, Gyntelberg F. Triglycerides concentrations and ischemic heart disease: an eight-year follow-up in the Copenhagen Male Study. Circulation 1998; 97: 1029-36

6. Jousilathi P, Tuomilehto J, Vartiainen E, Pekkanen J, Pusk P. Body weight, cardiovascular risk factors, and coronary mortality. 15-year follow-up of middle-aged men and women in eastern Finland. Circulation 1996; 93: 1372-79.

7. Sans S, Kestellot H, Kromhout D, on behalf of the Task Force. The burden of cardiovascular diseases mortality in Europe. Task Force of the European Society of Cardiology on cardiovascular mortality and morbidity statistics in Europe. Eur Heart J 1997; 18: 536-43.

8. Tunstall-Pedoe H, Kuulasmaa K, Mahönen M, Tolonen H, Ruokokoski E, Amouyel P, for the WHO MONICA project. Contribution of trends in survival and coronary-event rates to changes in coronary heart disease mortality: 10-year results from 37 WHO MONICA Project populations. Lancet $1999 ; 353: 1547-57$

9. Marrugat J, Solana P, D'Agostino R, Sullivan L, Ordovas J, Cordón F, Ramos R, Sala J, Masiá R, Rohlfs I, Elosua R, Kannel WB. Estimación del riesgo coronario en España mediante la ecuación de Framingham calibrada. Rev Esp Cardiol 2003; 56 : $253-261$

10. Yusuf HR, Giles WH, Croft JB, Anda RF, Casper ML. Impact of multiple risk factor profiles on determining cardiovascular disease risk. Prev Med 1998; $27: 1-9$
11. Wilson PW, Kannel WB, Sibershatz H, D’Agostino RB. Clustering of metabolic factors and coronary heart disease. Arch Intern Med 1999; 159: 1104-09.

12. Baena Díez JM, Álvarez Pérez B, Piñol Forcadell $P$, Martín Peñacoba R, Nicolau Sabaté M, Altès Boronat A. Asociación entre la agrupación (clustering) de factores de riesgo cardiovascular y el riesgo de enfermedad cardiovascular. Rev Esp Salud Pública 2002; 76:7-15.

13. Córdoba García R, Ortega Sánchez-Pinilla R, Cabezas Peña C, Forés García D, Nebot Adell M. Recomendaciones sobre el estilo de vida. Aten Primaria 1999; 24 (supl. 1): 118-32.

14. Programa de Actividades preventivas y de Promoción de la Salud. Guía de Prevención Cardiovascular. Madrid: Sociedad Española de Medicina Familiar y Comunitaria; 1996.

15. Programas básicos de salud. Diabetes Mellitus. Sociedad Española de Medicina Familiar y Comunitaria. Madrid: Doyma; 1998.

16. Ketola E, Sipila R, Makela M. Effectiveness of individual lifestyle interventions in reducing cardiovascular disease and risk factors. Ann Med 2000; 32: 239-51.

17. Evenson KR, Rosamond WD, Cai J, Toole JF, Hutchinson RG, Shahar E, et al. Physical activity and ischemic stroke. The atherosclerosis risk in communities study. Stroke 1999; 30: 1333-39.

18. Hu FB, Stamfer MJ, Colditz GA, Ascherio A., Rexrode KM, Willet WC, et al. Physical activity and risk of stroke in women. JAMA 2000; 283: 296167.

19. Hakim AA, Curb JD, Petrovitch H, Rodríguez BL, Yano K, Ross GW, et al. Effects of walking on coronary heart disease in elderly men: the Honolulu Heart Program. Circulation 1999; 100: 9-13. 20. Ebrahim S, Smith GD. Systematic review of randomised controlled trials of múltiple risk factors interventions for preventing coronary heart disease. BMJ 1997; 314: 1666-68.

21. Psaty BM, Smith NL, Siscovick DS, Koepsell TD, Weiss NS, Heckbert SR. Health outcomes associated with antihypertensive therapies used as firstline agents. A systematic review and meta-analysis. JAMA 1997; 277: 739-45.

22. Gould A, Rossouw JE, Santarello N, Heyse J; Furberg C. Cholesterol reduction yields clinical benefict: impact of statin trials. Circulation 1998; 97 : 946-52. 
23. Scheuermann W, Razum O, Scheidt, Wiesemann A, von Frankeberg L, Topf G, et al. Effectiveness of a decentralized, community-related approach to reduce cardiovascular disease risk factors levels in Germany. Eur Heart J 2000; 21: 1591-97.

24. Dielhl HA. Coronary risk reduction through intensive community-based lifestyle intervention: the Coronary Health Improvement Project (CHIP) experience. Am J Cardiol 1998; 82: 83-7.

25. Fortmann SP, Varady AN. Effects of a communitywide health education program on cardiovascular disease morbidity and mortality: the Stanford FiveCity Project. Am J Epidemiol 2000; 152: 316-23.

26. Kuulasmaa K, Tunstall-Pedoe H, Dobson A, Fortmann S, Sans S, Tolonen H, et al. Estimation of contribution of changes in classic risk factors to trends in coronary-event rates across the WHO MONICA Project populations. Lancet 2000; 355: 668-9.

27. Wilson PW, D'Agostino RB, Levy D, Belanger AM, Silbershatz H, Kannel WB. Prediction of coronary heart disease using risk factor categories. Circulation 1998; 97:1837-47.

28. Conroy RM, Pyöräla K, Fitzgerald AP, Sans S, Menotti A, De Backer G, et al. Estimation of tenyear risk of fatal cardiovascular disease in Europe: the SCORE project. Eur Heart J 2003; 24: 987 1003 .

29. United Kingdom Prospective Diabetes Study (UKPDS) 13: Relative efficacy of randomly allocated diet, sulphonylurea, insulin, or metformin in patients with newly diagnosed non-insulin dependent diabetes followed for three years. BMJ 1995; 310: $83-8$.

30. ALLHAT Officers and Coordinators for the ALLHAT Collaborative Research Group. The Antihypertensive and Lipid-Lowering Treatment to Prevent Heart Attack Trial. Major outcomes in moderately hypercholesterolemic, hypertensive patients randomized to pravastatin vs usual care: The Antihypertensive and Lipid-Lowering Treatment to Prevent Heart Attack Trial (ALLHAT-LLT). JAMA. 2002; 23:2998-3007. 